\title{
Achilles tendinosis is associated with sprouting of substance $P$ positive nerve fibres
}

\author{
T E O Schubert, C Weidler, K Lerch, F Hofstädter, R H Straub
}

Ann Rheum Dis 2005;64:1083-1086. doi: 10.1136/ard.2004.029876

\begin{abstract}
Objectives: To identify and characterise nerve fibres and inflammatory alterations in painful Achilles tendinosis and thus gain evidence about the origin of pain in Achilles tendinosis.

Methods: The composition of 10 tendon samples from patients with a prior history of painful Achilles tendinosis and 10 samples from patients with spontaneously ruptured tendons but no previous pain was compared by immunohistochemistry and conventional histology.

Results: The presence of granulation tissue was shown in 8/ 10 cases of Achilles tendinosis. Nociceptive substance P (SP) positive nerve fibres were significantly increased, and an inflammatory infiltration comprising B and T lymphocytes was found. Additionally, small foci with iron positive haemosiderophages, indicating prior microtraumatic events, were found in $6 / 10$ samples. None of the spontaneously ruptured tendons contained granulation tissue or haemosiderophages. Inflammatory infiltration in these patients consisted almost exclusively of granulocytes and SP positive nerve fibres were decreased. The density of sympathetic nerve fibres did not differ in the two conditions.

Conclusion: Achilles tendinosis is associated with the presence of granulation tissue, haemosiderophages, and SP positive nerve fibres, which may transmit the clinically pertinent pain. Achilles tendinosis may be caused by repeated microtraumata with ensuing organisation that is accompanied by sprouting of nociceptive SP positive nerve fibres.
\end{abstract}

\footnotetext{
A
} chilles tendinosis, also known as achillodynia, is viewed as a degenerative alteration of the Achilles tendon accompanied by pain and often associated with tendon thickening. It is common in athletes but occurs in non-athletes as well. ${ }^{1}$ Surgical specimens obtained from affected tendons show a range of degenerative changes such as changes in tendon fibre structure and arrangement as well as an increase in glycosaminoglycans, which may explain the swelling of the tendon. ${ }^{2}$ The pathophysiology of Achilles tendon pain is still unclear. Nociceptive nerve fibres can increase during inflammatory conditions-for example, in rheumatoid arthritis ${ }^{3}$ or in the course of wound healing, healing of fractures and other lesions..4

To elucidate one aspect of the pathophysiology of pain in Achilles tendinosis we examined the inflammatory infiltrate and innervation in 10 tendons from patients with Achilles tendinosis and 10 tendons from patients with spontaneous traumatic Achilles tendon ruptures.

\section{PATIENTS AND METHODS \\ Patients}

Ten Achilles tendon samples obtained from patients with Achilles tendinosis and 10 samples from patients with spontaneously ruptured Achilles tendons without a previous history of pain were submitted to the department of pathology between November 2000 and September 2003 for routine histological examination. The diagnostic criteria for Achilles tendinosis were tendon pain for several months and swelling of the tendon in the distal portion. Only spontaneously ruptured tendons which were resected within 48 hours after injury were included in the study. Table 1 shows the characteristics of the patients.

\section{Immunohistochemistry of inflammatory cells and iron staining}

Formalin fixed tissue was dehydrated and embedded in paraffin. Paraffin sections 3-5 $\mu \mathrm{m}$ thick were mounted on "Superfrost Plus" slides, heated for 20 minutes at $72^{\circ} \mathrm{C}$, deparaffinised, and rehydrated. For antigen retrieval, sections were placed in a microwave for 30 minutes at $240 \mathrm{~W}$ in a citrate buffer at $\mathrm{pH} 7.3$, then cooled to room temperature. After rinsing the slides, endogenous peroxidase activity was blocked by methanolic peroxide, the slides were rinsed again, and primary monoclonal antibody against CD3 for the detection of $\mathrm{T}$ lymphocytes, CD20 for $\mathrm{B}$ lymphocytes, or CD68 for macrophages (DAKO, Hamburg, Germany) in a dilution of 1:100 was applied. All slides were incubated using a Ventana machine and each antibody incubation was performed at $37^{\circ} \mathrm{C}$; a labelled streptavidin-biotin-peroxidase method at $37^{\circ} \mathrm{C}$ was used to visualise positive reaction (Ventana Medical Systems basic DAB detection kit, Ventana Medical Systems Inc, USA). To detect haemosiderophages, paraffin sections were stained by Turnbull's acid ferrocyanide reaction. Granulocytes were counted in haematoxylin and eosin stained sections prepared according to standard protocols.

The numbers of granulocytes, iron positive cells, CD3, CD20, and CD68 positive cells were evaluated in 10 randomly selected high power fields of view $(\times 400)$ and expressed per square millimetre.

\section{Immunohistochemistry of nerve fibres}

The determination of the substance $\mathrm{P}$ (SP) positive sensory nerve fibres and sympathetic tyrosine hydroxylase (TH) positive nerve fibres has been described previously. ${ }^{3}$ Deparaffinised and rehydrated sections were blocked and incubated overnight with primary antibodies against $\mathrm{TH}$ or SP (Chemicon, Temecula, CA, USA). Immunofluorescent staining was achieved using Alexa 546 conjugated secondary antibodies against mouse or rabbit IgG (Molecular Probes, Leiden, The Netherlands). The numbers of TH and SP positive nerve fibres were evaluated in 17 randomly selected high power fields of view $(\times 400)$ and expressed per square millimetre.

Abbreviations: $\mathrm{SP}$, substance $\mathrm{P} ; \mathrm{TH}$, tyrosine hydroxylase 
Table 1 Characteristics of the patients investigated in this study

\begin{tabular}{lll}
\hline Variable & $\begin{array}{l}\text { Tendon } \\
\text { rupture }\end{array}$ & Tendinosis \\
\hline Patients (M/F) & $8 / 2$ & $7 / 3$ \\
Age (years), mean (SD) & 48.0 & 47.3 \\
& $(13.8)$ & $(13.0)$ \\
Duration of pain before operation (months) & 0 & $6-120^{*}$ \\
$\begin{array}{l}\text { Patients who received paratendinous } \\
\text { corticosteroid injections (n) }\end{array}$ & 0 & 4 \\
$\begin{array}{l}\text { Patients with additional pathological } \\
\text { conditions (n) }\end{array}$ & $1 \dagger$ & $2 \ddagger$ \\
\hline
\end{tabular}

*Three patients reported pain for more than 12 months; the patient had hypertension; fone patient had hyperuricaemia without manifest gout, the second patient had calcium pyrophosphate dihydrate deposition disease (there were no calcium pyrophosphate dihydrate deposits visible in the tendon on microscopic examination).

\section{Statistical analysis}

The individual values of both groups were compared by Mann-Whitney test (SPSS V.11.0, SPSS Inc, Chicago, USA). Correlations were analysed using Spearman's rank correlation analysis (SPSS). A p value $<0.05$ was the significance level.
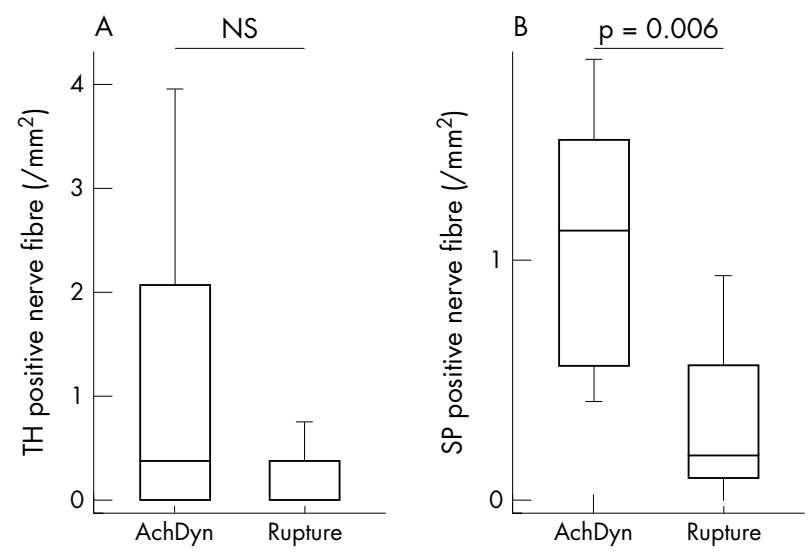

Figure 2 Comparison of sympathetic and nociceptive nerve fibres in painful tendinosis and ruptured tendon. The numbers of $\mathrm{TH}$ positive nerve fibres (A) and SP positive nerve fibres (B) were determined by immunofluorescent histochemistry in 10 tendons from patients with Achilles tendinosis (AchDyn) and 10 tendons from patients with spontaneously ruptured tendons. The number of nerve fibres was averaged from 17 high power fields and expressed as the number of fibres $/ \mathrm{mm}^{2}$. Box plots give the 10th, 25th, 75th, and 90th centiles. The median is given as a horizontal line within the box. SP, substance P; TH, tyrosine hydroxylase (sympathetic nerve fibres).
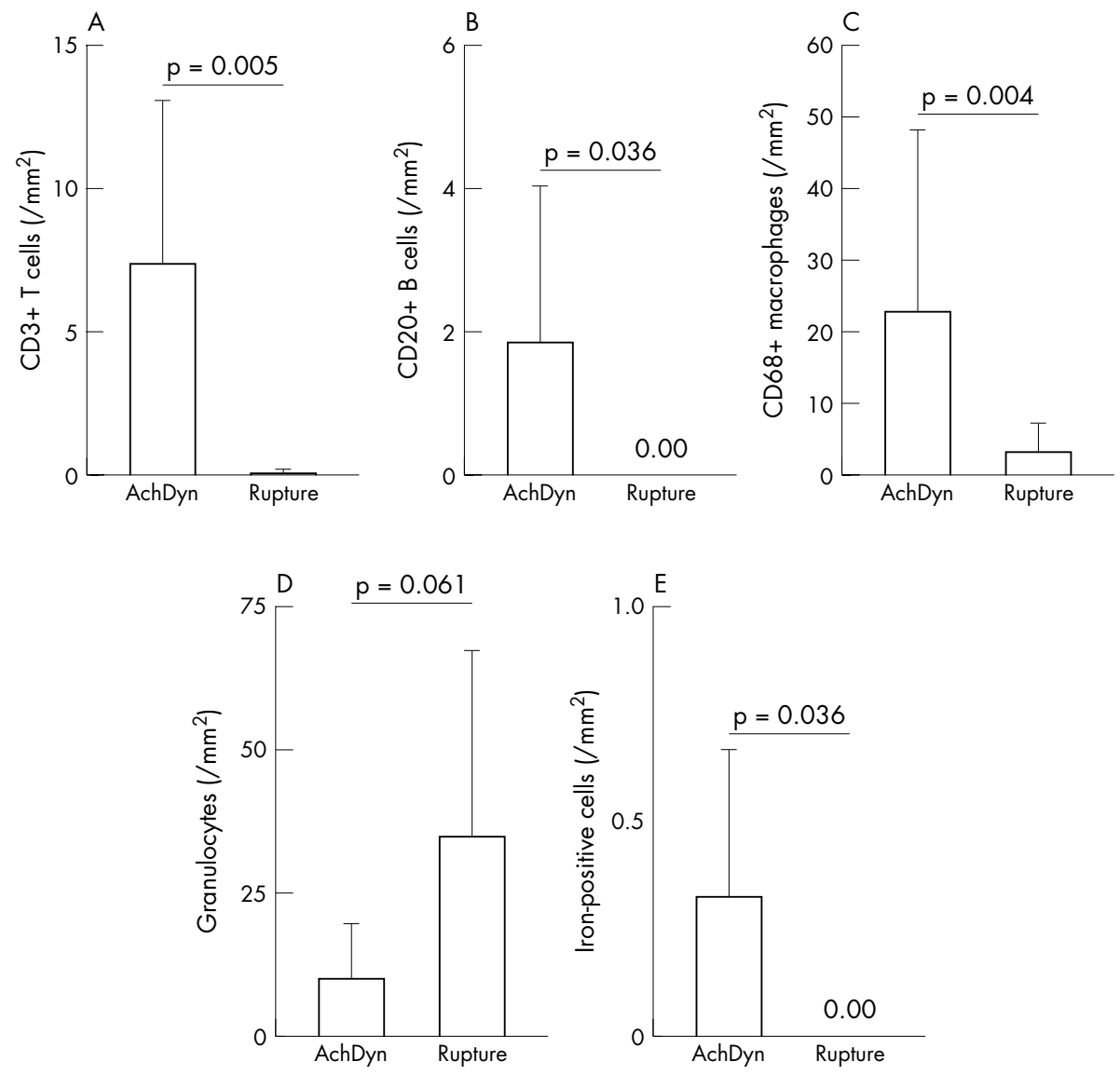

Figure 1 Determination of cell types in painful Achilles tendinosis and ruptured Achilles tendon. The numbers of (A) CD3+ and (B) CD20+ lymphocytes and (C) CD68+ macrophages were determined by immunohistochemistry in 10 tendons from patients with Achilles tendinosis and in 10 tendons from patients with spontaneously ruptured tendons. The number of (D) granulocytes was determined on haematoxylin and eosin stained sections and the number of (E) iron positive haemosiderophages was determined in sections stained by Turnbull's acid ferrocyanide reaction. The number of all cells was averaged from 10 high power fields and expressed as the number of cells $/ \mathrm{mm}^{2}$. AchDyn, painful Achilles tendinosis. 

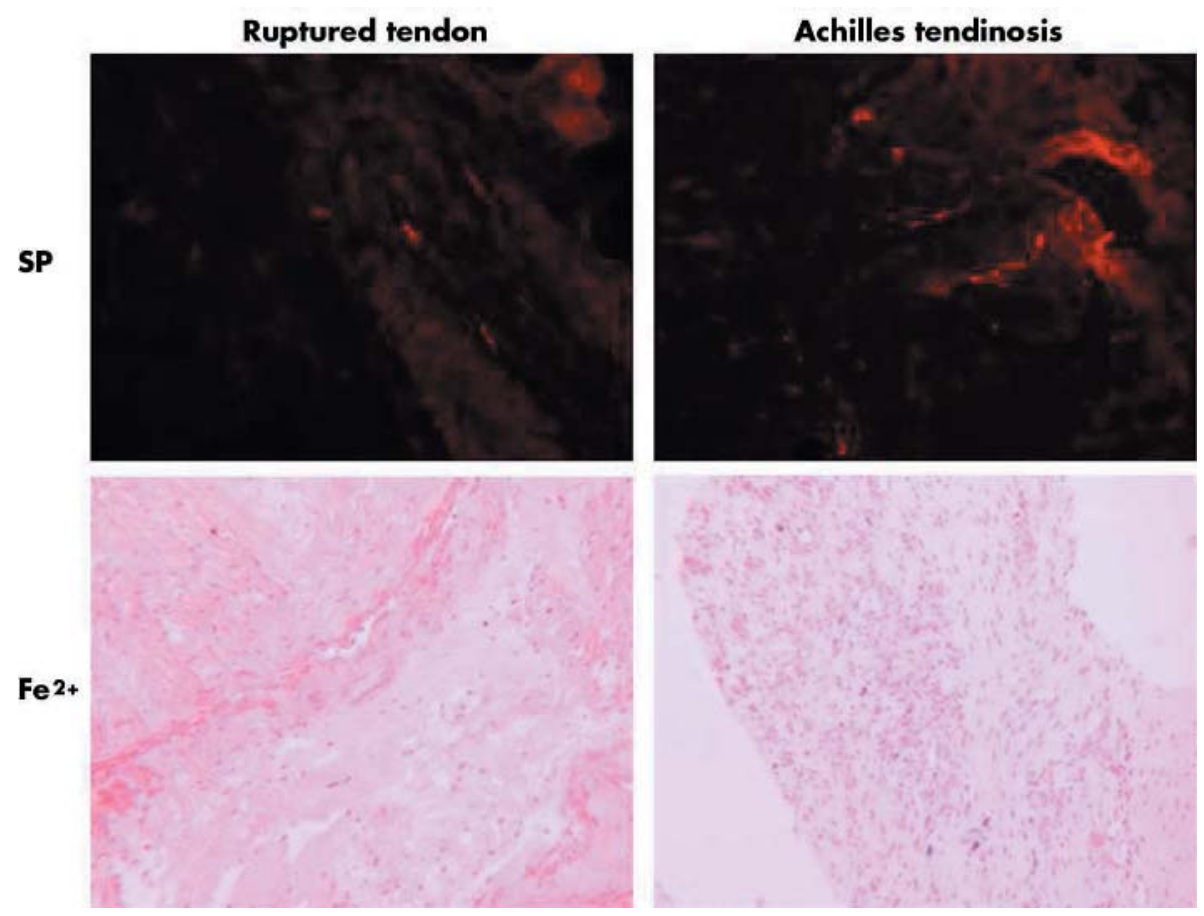

Figure 3 Histological sections of specimens from patients with ruptured tendons and from patients with tendinosis stained for substance $\mathrm{P}$ by immunofluorescence (SP, top row, original magnification $\times 400)$ and stained by Turnbull's acid ferrocyanide reaction to detect haemosiderophages $\left(\mathrm{Fe}^{2+}\right.$, bottom row, original magnification $\left.\times 5\right)$. Note that there are more SP positive nerve fibres visible in the tendinosis specimen than in the ruptured tendon. Haemosiderophages which are discernible as dark blue spots and small blood vessels were only found in the tendinosis specimen, not in the ruptured tendon.

\section{RESULTS}

Ten Achilles tendon samples obtained from patients with Achilles tendinosis and 10 samples from patients with spontaneously ruptured Achilles tendons without a previous history of pain were studied to characterise the innervation, inflammatory infiltration, presence of granulation tissue, and haemosiderophages. We found that the number of $\mathrm{B}$ lymphocytes, $\mathrm{T}$ lymphocytes, and macrophages was significantly higher in Achilles tendinosis samples (fig 1A-C), whereas the number of granulocytes tended to be higher in tissue from ruptured tendons (fig ID). Furthermore, in tendon samples from patients with Achilles tendinosis granulation tissue frequently containing haemosiderophages was found in 8/10 specimens (figs $\mathrm{IE}$ and 3). Granulation tissue consisted of clusters of capillaries embedded in a fibroblast-rich stroma infiltrated by macrophages, B lymphocytes, and $\mathrm{T}$ lymphocytes. None of the samples from spontaneously ruptured tendons contained granulation tissue or haemosiderophages (figs $1 \mathrm{E}$, and 3). The number of SP positive nerve fibres was significantly higher in tissue from patients with Achilles tendinosis than in tissue from patients with spontaneously ruptured tendons (figs 2 and 3). On average, 1.06 SP positive nerve fibres $/ \mathrm{mm}^{2}$ were found in the Achilles tendinosis group and 0.35 SP positive nerve fibres/ $\mathrm{mm}^{2}$ in ruptured tendons. The number of $\mathrm{TH}$ positive nerve fibres did not differ significantly between the two groups (fig 2).

The mean values for $\mathrm{TH}$ positive nerve fibres $/ \mathrm{mm}^{2}$ for the Achilles tendinosis group and for ruptured tendons were 1.00 and 0.18 , respectively.

In a correlation analysis in patients with tendinosis, T cells correlated positively with B cells (Spearman rank correlation coefficient $\left.\left(r_{\mathrm{s}}\right)=0.683, \mathrm{p}=0.020\right)$ and haemosiderophages $\left(r_{\mathrm{s}}=0.710, \mathrm{p}=0.014\right)$.

\section{DISCUSSION}

The origin of Achilles tendinosis has not been elucidated so far. Recently, neovascularisation in the affected tendon section correlating with pain was demonstrated by ultrasonography and magnetic resonance imaging. ${ }^{8-10}$ The finding was confirmed by immunohistochemistry, disclosing neovascularisation accompanied by nerve structures, which were not further characterised. ${ }^{8}{ }^{10}$

We compared the composition of 10 tendon samples from patients with a previous history of achillodynia with 10 samples from patients with spontaneously ruptured tendons without a previous history of pain by immunohistochemistry and conventional histology. Granulation tissue could be demonstrated in 8/10 cases of Achilles tendinosis. The granulation tissue comprised capillary vessels accompanied by an inflammatory infiltrate consisting of macrophages, $\mathrm{B}$ lymphocytes, and T lymphocytes. None of the spontaneously ruptured tendons contained granulation tissue or significant amounts of macrophages, B lymphocytes, and $\mathrm{T}$ lymphocytes. Instead they were infiltrated by large numbers of granulocytes as expected in the case of an acute traumatic event.

The number of nociceptive SP positive nerve fibres was found to be significantly higher in Achilles tendinosis samples than in tendon samples obtained from spontaneously ruptured tendons, whereas the number of $\mathrm{TH}$ positive nerve fibres did not differ. Spontaneously ruptured tendons were used as control group because immunohistochemistry for SP and $\mathrm{TH}$ in normal tendons taken from corpses did not deliver reproducible data. There are no published reports on the number of sensory nerve fibres of healthy human Achilles tendon. Both ruptured and Achilles tendinosis tendons contained low numbers of sensory nerve fibres compared with synovial tissue from healthy subjects, 
which contains 2.5 TH positive and 3.1 SP positive nerve fibres $/ \mathrm{mm}^{2}{ }^{21}$

The nerve fibres found in Achilles tendinosis specimens were often located next to small vessels in granulation tissue. Because we often found haemosiderophages within the granulation tissue, we suggest that microtraumatic events might have induced the formation of granulation tissue within the tendon. The degenerative alterations described in Achilles tendinosis may favour the occurrence of microtraumata. The growth of granulation tissue is accompanied by sprouting of sensory nerve fibres as demonstrated in animal models for wound healing. ${ }^{6}{ }^{7}$ Interestingly, no similar increase of sympathetic nerve fibres was found in our Achilles tendinosis specimens.

The pattern of organisation of experimentally ruptured rat Achilles tendons was studied by Ackermann et al. ${ }^{12}$ They found that uncompromised Achilles tendons from rats are devoid of nerve fibres, but after rupture of the tendon, extensive sprouting of nerve fibres into the tendon takes place during the healing phase. SP positive nerve fibres were observed in the emerging granulation tissue next to the rupture site 1 week after rupture and reached a maximum density at week 4 after rupture. In correlation, 1-4 weeks after rupture the nociception to thermal and mechanical stimuli increased. ${ }^{12}$ Because we obtained tendon samples very early after tendon rupture, no such sprouting phenomenon was seen in the ruptured Achilles tendons. With the completion of the regenerative process, SP positive nerve fibres withdrew from the tendon tissue 8 weeks after rupture. ${ }^{12}$ Assuming a similar time course of organisation, the simultaneous occurrence of granulation tissue, nerve fibres, and haemosiderophages accompanied by longstanding local pain in Achilles tendinosis indicates that repeated microtraumata might have occurred which maintain the presence of granulation tissue and SP positive nerve fibres.

Besides transmitting nociceptive signals, the sprouting sensory nerve fibres may have profound effects on tendon structure because it has been shown that SP stimulates the proliferation of fibroblasts ${ }^{13}$ and production of transforming growth factor $\beta$ by fibroblasts, ${ }^{14}$ potentially contributing to the thickening of the tendon seen in Achilles tendinosis.

To summarise, we found granulation tissue, haemosiderophages and, SP positive nerve fibres in tendons affected by Achilles tendinosis. The nociceptive nerve fibres may transmit the clinically pertinent pain. We suggest that Achilles tendinosis may be caused by repeated microtraumata with the ensuing organisation that is accompanied by sprouting of nociceptive SP positive nerve fibres.

\section{ACKNOWLEDGEMENTS}

Supported by the DFG (Str 511/10-1) and the University of Regensburg funding programme ReForM C.

\section{Authors' affiliations}

T E O Schubert, F Hofstädter, Department of Pathology, University of

Regensburg, Regensburg, Germany

C Weidler, R H Straub, Department of Internal Medicine I, University of

Regensburg, Regensburg, Germany

K Lerch, Department of Orthopaedic Surgery, University of Regensburg, Bad Abbach, Germany

T E O Schubert and C Weidler contributed equally to the study.

Correspondence to: Dr T E O Schubert, Department of Pathology, Franz-Josef-Strauss-Allee 11, 93053 Regensburg, Germany;

thomas.schubert@klinik.uni-regensburg.de

Accepted 15 December 2004

\section{REFERENCES}

1 Rolf C, Movin T. Etiology, histopathology, and outcome of surgery in achillodynia. Foot Ankle Int 1997; 18:565-9.

2 Movin T, Gad A, Reinholt FP, Rolf C. Tendon pathology in long-standing achillodynia. Biopsy findings in 40 patients. Acta Orthop Scand 1997;68:170-5.

3 Miller LE, Justen HP, Scholmerich J, Straub RH. The loss of sympathetic nerve fibers in the synovial tissue of patients with rheumatoid arthritis is accompanied by increased norepinephrine release from synovial macrophages. FASEB J 2000;14:2097-107.

4 Wolf K, Hocherl E, Farkas T, Pfister C. [Expression and dynamics of peptidergic nerve fibers in granulation tissue after distance osteosynthesis. ] Unfallchirurg, 2001;104:948-54.

5 Walsh DA, Hu DE, Mapp PI, Polak JM, Blake DR, Fan TP. Innervation and neurokinin receptors during angiogenesis in the rat sponge granuloma. Histochem J 1996;28:759-69.

6 Dunnick CA, Gibran NS, Heimbach DM. Substance P has a role in neurogenic mediation of human burn wound healing. J Burn Care Rehabil 1996; 17:390-6.

7 Lai X, Wang Z, Wei L, Wang L. Effect of substance $P$ released from peripheral nerve ending on endogenous expression of epidermal growth factor and its receptor in wound healing. Chin J Traumatol 2002;5:176-9.

8 Shalabi A, Kristoffersen-Wiberg M, Papadogiannakis N, Aspelin P, Movin T. Dynamic contrast-enhanced MR imaging and histopathology in chronic achilles tendinosis. A longitudinal MR study of 15 patients. Acta Radiol 2002;43:198-206.

9 Zanetti M, Metzdorf A, Kundert HP, Zollinger H, Vienne P, Seifert B, et al. Achilles tendons: clinical relevance of neovascularization diagnosed with power Doppler US. Radiology 2003;227:556-60.

10 Alfredson $\mathrm{H}$, Ohberg L, Forsgren $\mathrm{S}$. Is vasculo-neural ingrowth the cause of pain in chronic Achilles tendinosis? An investigation using ultrasonography and colour Doppler, immunohistochemistry, and diagnostic injections. Knee Surg Sports Traumatol Arthrosc 2003;11:334-8.

11 Miller LE, Weidler C, Falk W, Angele P, Schaumburger J, Scholmerich J, et al. Increased prevalence of semaphorin 3C, a repellent of sympathetic nerve fibers, in the synovial tissue of patients with rheumatoid arthritis. Arthritis Rheum 2004;50:1156-63.

12 Ackermann PW, Li J, Lundeberg T, Kreicbergs A. Neuronal plasticity in relation to nociception and healing of rat achilles tendon. J Orthop Res 2003;21:432-41.

13 Katayama I. Nishioka K. Substance P augments fibrogenic cytokine-induced fibroblast proliferation: possible involvement of neuropeptide in tissue fibrosis, J Dermatol Sci 1997; 15:201-6.

14 Lai XN, Wang ZG, Zhu JM, Wang LL. Effect of substance P on gene expression of transforming growth factor beta- 1 and its receptors in rat's fibroblasts. Chin J Traumatol 2003;6:350-4. 